\title{
Effect of Tai Chi Chuan training on main performance during dance movements.
}

\author{
Hai-Bin Yu ${ }^{1,2 \#}$, Yi-Hsin Chang ${ }^{3 \#}$, Pao-Yi Liao ${ }^{3}$, Yung-Shen Tsai ${ }^{*}$ \\ ${ }^{1}$ Institute of Sports Sciences, University of Taipei, Taipei, Taiwan \\ ${ }^{2}$ School of Physical Education, Quanzhou Normal University, Quanzhou, PR China \\ ${ }^{3}$ Department of Dance, University of Taipei, Taipei, Taiwan \\ ${ }^{4}$ Institute of Sports Equipment Technology, University of Taipei, Taipei, Taiwan \\ \#These authors have equally contributed to this study.
}

\begin{abstract}
Purpose: Tai Chi Chuan (TCC) training is usually considered as useful training methods for improving postural stability during human movement performance. Whether the jumps, spin, and balance required in dance movements can be reinforced through TCC training is not certain. This study was to investigate the influence of 8-week TCC training on the selected dance movements.

Method: A total of 16 senior high school dancers participated in this study (8 in the training group and 8 in the control group). A force platform and a 3D motion analysis system were used to analyze the movements of sauté (jump), passé (balance), and pirouette en dehors (spin).

Results: The results showed that the training group demonstrated greater improvement than did the control group in the following areas: (a) The maximum lower extremity push-off force and air hang time during sauté jumps; (b) The center of gravity (COG) shift distance and shift speed during passé balance; (c) The working leg hip joint external rotation angle, the supporting leg plantar flexion angle, and the COG shift distance during pirouette en dehors spin.

Conclusion: TCC training facilitated reinforcing dance movements and may be used as part of physical training program for improving dance performance.
\end{abstract}

Keywords: Tai chi chuan training, COG shift, Maximum lower extremity push-off force, Balance, Jump, Spin performance during dance movements, Two-way mixed ANOVA analysis.

Accepted on April 16, 2018

\section{Introduction}

During dance performance, dancers must favourably coordinate their fundamental abilities, including flexibility, balance, postural stability, muscle strength and power, with the rhythm of the music to enable their bodies presenting the most perfect movements on stage [1,2]. Dancers need to have desirable flexibility to perform techniques with large body movement. They must rely on their muscle strength as well as balance and postural stability to complete complex movement combinations. Moreover, having appropriate muscle strength and power is relatively important for performing jumps, spin, and other highly difficult movements.

Several dance companies in Taiwan have introduced Tai Chi Chuan (TCC) training for dancers to improve their physical ability and performance. The concept of TCC training is considered to be able to improve dancer's body control and awareness. Research has reported that TCC training facilitates improving muscle strength and flexibility as well as balance and postural stability [3-9]. Lan et al. found that TCC training increased knee extensor muscle strength and endurance [10]. $\mathrm{Li}$ et al. found that knee flexor muscle strength and reaction time were improved after TCC training [8]. Guo et al. found that individuals who practiced TCC for many years performed better in balance, ankle proprioception, and knee muscle strength tests than those who did not practice TCC [4]. These improvements may contribute to movement stability. Zheng et al. examined effectiveness of 12-week TCC training on physical health of college students and found that their sit and reach flexibility and balance ability were improved [9]. Webster et al. did a systematic review including 76 studies regarding the health benefits of TCC for college students. They found that possible benefits of TCC practice comprise increased flexibility, improved balance, and improved $800 / 1000$ meter run time [8]. However, whether such improvement further enhances jump, spin, and balance movements in dance performance is not certain. 
On the basis of the aforementioned findings, both dance and TCC performance require superior whole body coordination, balance, body weight shift, and lower extremity mobility. TCC training has been reported to be useful for enhancing human movement ability. Whether TCC training can facilitate improving the movement performance of dancers was, therefore, the aim of the study. We hypothesized that dancers who receive an 8-week TCC training would improve their movement performance in jump, balance, and spin.

\section{Methods}

\section{Participants}

The study participants were high school dance class female students who had no previous experience of TCC training. The participants were divided into a training group and a control group with 8 students in each group. All participants came from the same class and had all received identical dance lessons (Table 1). The training group received TCC training for 8 weeks. The control group did not participate in any other physical training during the same period of time. None of the participants experienced injuries or any neurological or musculoskeletal diseases during the training period and within the 6 months preceding the start of the study. Before the study, informed consent was obtained from both the participants and their parents. The study was approved by the University Institutional Review Board.

Table 1. Comparison of the demographic information between the two groups.

\begin{tabular}{lllc}
\hline Parameter & Training group $(\mathbf{n}=8)$ & $\begin{array}{l}\text { Control } \\
(\mathbf{n}=8)\end{array}$ & group \\
\hline Age $(\mathrm{y})$ & $17.3 \pm 0.5$ & $17.3 \pm 0.5$ & 1 \\
\hline Height $(\mathrm{cm})$ & $159.4 \pm 4.4$ & $159.4 \pm 2.6$ & 0.814 \\
\hline Weight $(\mathrm{kg})$ & $48.1 \pm 3.4$ & $50.7 \pm 3.7$ & 0.166 \\
\hline Dance experience $(\mathrm{y})$ & $10.5 \pm 1.7$ & $10.5 \pm 2.3$ & 1 \\
\hline
\end{tabular}

\section{Tai chi chuan training}

The TCC training program used in this study is the Yang-style 24-form TCC designated for competition by the International Wushu Federation (IWUF). The training program lasted 8 weeks, with two sessions per week and each session lasting 1.5 h. Each 1.5-h training session included warm-up, basic skills practice, cool-down, and breathing relaxation exercises.

\section{Procedures}

During each of the testing sessions, the participants wore their own dance costumes, safety shorts, and dance shoes. They performed $15 \mathrm{~min}$ of warm-up activities, including activities for the various joints and whole-body stretching, and practiced movements for the test. After the warm-up, each participant's body weight was measured for subsequent force platform data standardization. The Sauté jumps and the Passé balance were measured with an AMTI force platform (Advanced Mechanical Technology, Inc., Watertown, MA, USA) at the frequency of 1,000 Hz. A 3D motion capture and analysis system (Raptor-E Digital RealTime System, Motion Analysis Corporation, Santa Rosa, CA, USA) with 9 infrared cameras $(200 \mathrm{~Hz})$ was synchronized with the force platform for Pirouette en dehors spin motion analysis. A total of 13 reflective markers were attached to each participant's body at the following locations: sacrum, bilateral anterior superior iliac spine, middle of right thigh and left thigh at the lateral side, lateral epicondyle of right and left knee joint, middle of right shank and left shank at the lateral side, right and left ankle malleolus, right and left second metatarsal head. Three movement tests were conducted as the following sequence: (a) Sauté jump test: the participants stood on the force platform and performed the saute jump when they were ready. They performed one jump at a time for a total of three times with 1 min of rest between jumps; (b) Passé balance test: after the participants stood on their supporting leg and maintained balance on their own, the test was timed to $5 \mathrm{~s}$. The test was repeated for a total of three times with one minute of rest between trials; (c) Pirouette en dehors spin test: the participants stood on the force platform and performed the pirouette en dehors movement. They executed two revolutions each trial and performed five trials with 1 min of rest between trials. Three most stable trials were selected for data analysis. Each participant had 5-10 min rest between each of the 3 types of testing. After the training group finished 8-week TCC training program, both groups of participants engaged in the post-training test using the same aforementioned methods.

\section{Data analysis}

The maximum lower extremity push-off force during the sauté jump and the trajectory coordinates of center of gravity (COG) during passé balance and pirouette en dehors spin were derived from the force platform data using Cortex software (Motion Analysis Corporation, Santa Rosa, CA, USA). The anteroposterior (AP) and mediolateral (ML) COG shift distance and average COG shift speed were calculated for statistical analysis.

For getting working leg hip joint external rotation angle and supporting leg plantar flexion angle during pirouette en dehors spin, coordinate data of all reflective markers were exported from the Cortex software and then imported into the OrthoTrak software (Motion Analysis Corporation, Santa Rosa, CA, USA) for motion analysis. The air hang time during sauté jump spanned from the take-off time when the toes left the ground to the moment the feet landed on the ground again. Specifically, the take-off time pertained to when the difference in the elevation of the reflective marker on the second metatarsal bone exceeded two standard deviations. Through a similar method, the landing time was derived from the elevation of the reflective marker on the instep after landing. 


\section{Statistical analyses}

The independent t test was used to compare the demographic information of the participant groups. The effect of training was tested using a $2 \times 2$ two-way mixed analysis of variance (ANOVA) design for each outcome variable, with time (pre and post-training test) as within subject factor and group as between subject factor. If there was an interaction between the two factors on an outcome variable, the paired t-test then was performed to compare the differences between pre- and posttraining test for each group and the independent t-test was performed to compare group differences for each of pre and post-training test. In addition, independent t-tests were also used to compare the magnitude of improvement in each outcome variable between groups. Cohen's d was calculated using the means and standard deviations of the two groups to determine the effect size of comparisons [11]. SPSS 12.0 (SPSS $^{\circledR}$, Chicago, IL, USA) was used for data analysis. Statistical significance was set at $\mathrm{p}<0.05$.

\section{Results}

There were no significant differences in the demographic data between the two groups. The age, height, weight, and dance experience of the two groups of participants are listed in Table 1.

Two-way mixed ANOVA for maximum lower extremity pushoff force during sauté jump revealed that there were no main effects of time $(p=0.121)$ and group $(p=0.106)$, but the time $\times$ group interaction effect was significant $(p=0.001)$. Paired $t$ tests indicated that post-test was significantly greater than pretest in the training group $(p=0.002)$, but not in the control group $(p=0.126)$. Independent $t$-tests indicated that the training group was less than the control group in the pre-test $(p=0.017)$, but there was no significant difference between the two groups in the post-test $(\mathrm{p}=0.555)$.

Two-way mixed ANOVA for air hang time during sauté jump revealed that there were no main effects of time $(p=0.256)$ and group $(p=0.645)$, but the time $\times$ group interaction effect was significant $(\mathrm{p}<0.001)$. Paired t tests indicated that post-test was significantly longer than pre-test in the training group $(p<0.001)$, but post-test was significantly shorter than pre-test in the control group $(\mathrm{p}=0.003)$. Independent $\mathrm{t}$-tests indicated that there were no significant differences between the two groups in both pre-test $(\mathrm{p}=0.432)$ and post-test $(\mathrm{p}=0.109)$.

Table 2 illustrates the comparison of the change amplitude in the maximum lower extremity push-off force and air hang time during sauté jump after TCC training between the two groups. The results revealed that the change amplitude in the lower extremity push-off force during sauté jumps of the training group was significantly greater than that of the control group $(\mathrm{p}<0.001)$. Moreover, the change amplitude in the sauté jump hang time of the training group was also significantly greater than that of the control group $(\mathrm{p}<0.001)$.

Table 2. Comparison of the change amplitude in the maximum lower extremity push-off force and air hang time during sauté jump after Tai Chi Chuan training between the two groups.

\begin{tabular}{|c|c|c|c|c|c|c|}
\hline Parameter & Group & Pre-test & Post-test & $\begin{array}{l}\text { Pre-test-post-test } \\
\text { difference }(\%)\end{array}$ & $\mathbf{p}$ & Cohen's d \\
\hline \multirow{2}{*}{$\begin{array}{l}\text { Maximum lower extremity } \\
\text { push-off force }(\mathrm{N})\end{array}$} & Training & $1021.44 \pm 50.47$ & $1068.45 \pm 55.43$ & $4.63 \pm 2.86$ & \multirow{2}{*}{$<0.001^{\star}$} & \multirow{2}{*}{2.258} \\
\hline & Control & $1108.44 \pm 75.15$ & $1087.37 \pm 68.95$ & $-1.84 \pm 2.87$ & & \\
\hline \multirow{2}{*}{ Air hang time (s) } & Training & $1.85 \pm 0.16$ & $1.95 \pm 0.13$ & $5.18 \pm 2.65$ & \multirow{2}{*}{$<0.001^{*}$} & \multirow{2}{*}{3.170} \\
\hline & Control & $1.93 \pm 0.19$ & $1.79 \pm 0.22$ & $-7.03 \pm 4.76$ & & \\
\hline
\end{tabular}

Note: ${ }^{*} p<0.001$ indicates a significant difference between the pre-test-post-test differences of the two groups.

Two-way mixed ANOVA for anteroposterior COG shift distance during passé balance revealed that there was a main effect of time $(\mathrm{p}=0.015)$, but there was no main effect of group $(p=0.167)$. The time $\times$ group interaction effect was significant $(p=0.034)$. Paired $t$ tests indicated that post-test was significantly shorter than pre-test in the training group $(p=0.015)$, but both pre-test and post-test were similar in the control group $(p=0.724)$. Independent $t$-tests indicated that there were no significant differences between the two groups in both pre-test $(\mathrm{p}=0.062)$ and post-test $(\mathrm{p}=0.815)$.

Two-way mixed ANOVA for mediolateral COG shift distance during passé balance revealed that there was a main effect of time $(p=0.012)$, but there was no main effect of group $(\mathrm{P}=0.498)$. The time $\times$ group interaction effect was significant $(p=0.008)$. Paired t-tests indicated that post-test was significantly shorter than pre-test in the training group $(\mathrm{p}=0.005)$, but both pre-test and post-test were similar in the control group $(\mathrm{p}=0.889)$. Independent t-tests indicated that there were no significant differences between the two groups in both pre-test $(\mathrm{p}=0.098)$ and post-test $(\mathrm{p}=0.254)$.

Two-way mixed ANOVA for COG shift speed during passé balance revealed that there were no main effects of time $(p=0.691)$ and group $(p=0.584)$, but the time $\times$ group interaction effect was significant $(p<0.001)$. Paired $t$ tests indicated that post-test was significantly slower than pre-test in the training group $(p=0.043)$, but post-test was significantly faster than pre-test in the control group $(p=0.003)$. Independent $t$ tests indicated that the training group was slower than the control group in pre-test $(\mathrm{p}=0.007)$, but both groups were not significantly different in post-test $(\mathrm{p}=0.120)$. 
Table 3 compares the change amplitude regarding the AP and ML COG shift distance and average COG shift speed during passé balance after TCC training between the two groups. The results revealed that the magnitude of reduction in the AP COG shift distance of the training group was significantly greater than that of the control group $(p=0.040)$. The magnitude of reduction in the ML COG shift distance of training group was also significantly greater than that of the control group $(p=0.008)$. Finally, the magnitude of reduction in the average COG shift speed of the training group was significantly higher than that of the control group ( $p=0.001)$.

Table 3. Comparison of the change amplitude in the COG shift distances and speeds during passé balance after Tai Chi Chuan training between the two groups.

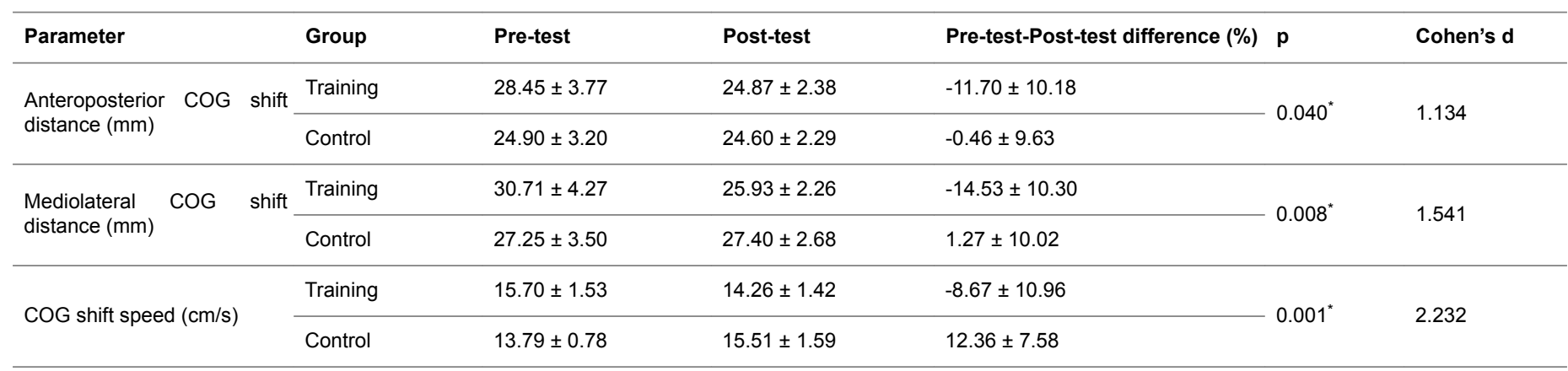

Note: ${ }^{*} p<0.05$ indicates a significant difference between the pre-test-post-test differences of the two groups.

Two-way mixed ANOVA for external rotation angle of the working leg hip joint during pirouette en dehors spin revealed that there was a main effect of time $(p=0.025)$, but there was no main effect of group $(\mathrm{p}=0.241)$. The time $\times$ group interaction effect was significant $(p=0.001)$. Paired tests indicated that post-test was significantly greater than pre-test in the training group $(p=0.006)$, but there was no significant difference between pre-test and post-test in the control group $(p=0.150)$. Independent t-tests indicated that the training group was significantly smaller than the control group in pre-test $(p=0.029)$, but there was no significant difference between the two groups in post-test $(\mathrm{p}=0.150)$.

Two-way mixed ANOVA for supporting leg plantar flexion angle during pirouette en dehors spin revealed that there were no main effects of time $(p=0.728)$ and group $(p=0.187)$, but the time $x$ group interaction effect was significant $(p=0.001)$. Paired t-tests indicated that post-test was significantly greater than pre-test in the training group $(\mathrm{p}<0.001)$, but pre-test and post-test were not significantly different in the control group $(p=0.057)$. Independent $t$-tests indicated that there was no significant difference between the two groups in pre-test $(p=0.645)$, but the training group was significantly greater than the control group in post-test $(\mathrm{p}=0.011)$.

Two-way mixed ANOVA for anteroposterior COG shift range during pirouette en dehors spin revealed that there were no main effects of time $(p=0.398)$ and group $(p=0.225)$, but there was a time $x$ group interaction effect $(p=0.014)$. Paired t-tests indicated that post-test was significantly shorter than pre-test in the training group $(\mathrm{p}=0.009)$, but pre-test and post-test were not significantly different in the control group $(p=0.298)$.
Independent $\mathrm{t}$ tests indicated that there was no significant difference between the two groups in pre-test $(p=0.477)$, but the training group was significantly shorter than the control group in post-test $(\mathrm{p}=0.027)$.

Two-way mixed ANOVA for mediolateral COG shift range during pirouette en dehors spin revealed that there were no main effects of time $(p=0.220)$ and group $(p=0.369)$, but there was a time $\times$ group interaction effect $(p=0.003)$. Paired t-tests indicated that post-test was significantly shorter than pre-test in the training group $(\mathrm{p}=0.003)$, but pre-test and post-test were not significantly different in the control group $(p=0.228)$. Independent $\mathrm{t}$ tests indicated that there was no significant difference between the two groups in pre-test $(p=0.135)$, but the training group was significantly shorter than the control group in post-test $(\mathrm{p}=0.004)$.

Table 4 presents comparison of the change amplitude in the working leg hip joint external rotation angle, supporting leg plantar flexion angle, and AP and ML COG shift distance during pirouette en dehors spin after TCC training between the two groups. The results showed that the magnitude of improvement in the working leg hip joint external rotation angle of the training group was significantly greater than that of the control group ( $p=0.001)$. The magnitude of improvement in the supporting leg plantar flexion angle of the training group significantly exceeded that of the control group $(p=0.001)$. The magnitude of improvement in the AP COG shift distance in the training group was significantly greater than that of the control group $(p=0.024)$. Finally, the magnitude of improvement in the ML COG shift distance of the training group was significantly greater than that of the control group $(\mathrm{p}=0.009)$.

Table 4. Comparison of the change amplitude in the working leg hip joint external rotation angle, supporting leg plantar flexion angle, and COG shift distance during pirouette en dehors spins after TCC training between the two groups. 


\begin{tabular}{|c|c|c|c|c|c|c|}
\hline Parameter & Group & Pre-test & Post-test & $\begin{array}{l}\text { Pre-test-Post-test } \\
\text { difference }(\%)\end{array}$ & $\mathbf{p}$ & Cohen's d \\
\hline \multirow{2}{*}{$\begin{array}{l}\text { External rotation angle of the } \\
\text { working leg hip joint (degrees) }\end{array}$} & Training & $117.57 \pm 7.83$ & $124.89 \pm 6.97$ & $6.38 \pm 4.79$ & $0.001^{*}$ & 2.033 \\
\hline & Control & $126.41 \pm 6.68$ & $124.57 \pm 7.64$ & $-1.47 \pm 2.62$ & & \\
\hline \multirow{2}{*}{$\begin{array}{l}\text { Supporting leg plantar flexion } \\
\text { angle (degrees) }\end{array}$} & Training & $131.90 \pm 4.67$ & $135.55 \pm 5.39$ & $2.76 \pm 1.14$ & $0.001^{*}$ & 2.036 \\
\hline & Control & $132.97 \pm 4.45$ & $128.61 \pm 4.06$ & $-3.20 \pm 3.98$ & & \\
\hline \multirow{2}{*}{$\begin{array}{l}\text { Anteroposterior COG shift range } \\
(\mathrm{mm})\end{array}$} & Training & $489.44 \pm 143.39$ & $354.12 \pm 56.58$ & $-24.39 \pm 14.54$ & $0.024^{*}$ & 1.263 \\
\hline & Control & $448.74 \pm 98.69$ & $519.76 \pm 180.40$ & $17.03 \pm 44.07$ & & \\
\hline \multirow{2}{*}{$\begin{array}{l}\text { Mediolateral COG shift range } \\
(\mathrm{mm})\end{array}$} & Training & $500.93 \pm 93.68$ & $338.07 \pm 85.60$ & $-31.77 \pm 15.35$ & $0.009^{*}$ & 1.519 \\
\hline & Control & $414.98 \pm 121.27$ & $490.95 \pm 90.67$ & $29.59 \pm 55.04$ & & \\
\hline
\end{tabular}

Note: ${ }^{*} p<0.05$ indicates a significant difference between the pre-test-post-test differences of the two groups.

\section{Discussion}

The purpose of this study was to investigate whether TCC training facilitates improving the jump, balance, and spin movements of dancers. The results found that the improvement of the training group in each movement test was significantly greater than that of the control group. These results are discussed in the following sections.

The study showed that after 8 weeks of TCC training, improvement in the training group regarding the maximum lower extremity push-off force and air hang time were significantly greater than that of the control group. This might be attributable to how TCC movements emphasize the use and coordination of the lower extremities. Particularly, performing horse stance and sustaining a half-squat posture for an extensive period during TCC training may facilitate increasing muscle strength in the lower extremities. The mechanism of power generation in TCC originates from the feet, followed by contracting the gluteus maximus, thus extending the hip joint. This may enable generating a downward force through stomping the ground, from which a ground reaction force propagates upward [12-14]. This concept, which involves force generation through alternative sources and the synchronous use of lower trunk and legs, may be applied to dance instruction. The overall coordination of the core muscles, upper extremities, and lower extremities is essential to dancers performing jumps. TCC training may facilitate controlling core muscles and coordinating the upper and lower extremities. Principal dancers' jump and spin techniques are demonstrations of specific dance movements. These movements not only involve the technical aspects of dancing but also require dancers to maintain an aesthetic posture and temperament when performing jumps $[15,16]$. Dance pertains to a type of art instead of a high jump sport. A dancer's most brilliant and artistic skill involves creating a temporary illusion of halting in mid-air. An extensive air time enables a dancer to create such an illusion while expressing a feeling of lightness [5]. This phenomenon may be benefit from TCC training that is able to facilitate enhancing the maximum lower extremity push-off force during sauté jump and extending air hang time in this study. In addition, each jump movement in ballet is an extension of the basic sauté. Dancers with a favourable sauté foundation can favourably perform advanced techniques.

Balance ability is a crucial physical ability for dancers who need to stand on a stage and pursue the most perfect performance. This study showed that after TCC training, the improvement in the AP COG shift distance, ML COG shift distance, and COG shift speed of the training group during passé balance were significantly greater than those of the control group. This result accords with those of previous studies that the TCC training group exhibited improved balance ability $[3,4,9,17]$. Xu et al. demonstrated that longterm TCC training markedly affects knee and ankle joint proprioception [18]. They also indicated that TCC training improved body control ability more than long-term running could. Thus, TCC practice might facilitate improving dancers' ankle and knee joint stability. The better the body's ability to balance is, the easier and more accurately can the weight be made to fall on the strongest sites of the legs. In other words, dancers must master to correctly manage their body COG in order to balance their bodies and distribute force evenly in the supporting leg, eliminate unnecessary physical tension, thus reducing the pressure and damage to the load-bearing parts of the body. Mak et al. asserted a close correlation between TCC and body control [7]. Individuals who practiced TCC attained better results than those who had not practiced TCC in both clinical and laboratory tests. Overall, the aforementioned research indicates that TCC training may facilitate learning how to relax the entire body and effectively shifting weight between the two feet. This enables sustaining balance when standing on one leg, with the trunk straight and centred. If the lower extremities stably support the entire body, the upper extremities can be agilely used, generating synchronous effect complementing movement and stillness, virtuality and reality, and rigidity and flexibility. These are the goals that ballet training must attain.

Only with the support of favourable and stable balance ability can dancers freely perform superb spin techniques. Dancers with sufficient stability do not need to shift their position or adjust their posture at the conclusion of a spin or large jump 
movement. In this study, the improvement in the AP COG shift distance and ML COG shift distance of the training group during Pirouette en dehors performance were significantly greater than those of the control group. This might be attributable to how TCC emphasizes COG shifting. Regardless of shifting from 2 feet stance to a one-foot stance or an alternative shift between standing on the either support foot, such exercises cause dancers to experience a constant change in the COG and enhance their supporting leg balance ability to support whole-body spin movements. A previous study revealed that 12-week strength training for hamstring and quadriceps muscles improved dancer's balance ability, but their rotation ability improvement was inconsistent, indicating that they might need additional practice of timing with the head, arm, and leg movements [2]. TCC training may provide the following benefits to dancers: understanding the effective use of the body central axis and force, increasing the mastery of the COG management and movement stability, improving the body's perception of time and space, and enhancing attention $[4,5,19]$. Therefore, the training group in the present study might be able to control their bodies more easily and exhibited favourable spatial perception in their bodies, thus enabling them to perform stable spin movements. In addition, the improvement in the working leg hip joint external rotation angle was superior to that of the control group. This result might be attributable to how the supporting leg of the training group possessed relatively better stability to support balance, enabling the working leg to attain a relatively better external opening angle during rotation. If the supporting leg does not possess the ability to stabilize the body, the working leg cannot attain a favourable external opening angle, and attaining a clean, beautiful, and correct rotation becomes relatively difficult. A review study also reported that physical training in addition to traditional dance training may contribute to dancer's movement range of motion [20,21]. Finally, the improvement in the supporting leg plantar flexion angle of the training group was superior to that of the control group. This difference might be because balance ability in the supporting leg of the training group was enhanced, enabling them to support the body higher while spinning. Future research can continue investigating whether the external rotation angle of the working leg hip joint and the supporting leg plantar flexion angle influence rotation quality.

\section{Conclusion}

The study showed that if high school dance class students participate in an additional 8 weeks of TCC training, their dance performance can be enhanced. The participants in the training group also felt that such training is beneficial for their body coordination and postural stability. If dancers can experience the sensations of slowness, calmness, and relaxation regarding TCC and then apply these sensations to achieve swift, sharp, and stable dance moves, they can adequately adjust the speeds of their bodies, facilitating their dance performance.

\section{Acknowledgment}

This work was partially supported by the grant from the University of Taipei.

\section{References}

1. Hamilton LH, Hamilton WG, Warren MP, Keller K, Molnar M. Factors contributing to the attrition rate in elite ballet students. J Dance Med Sci 1997; 1: 131-138.

2. Koutedakis Y, Sharp NC. Thigh-muscles strength training, dance exercise, dynamometry, and anthropometry in professional ballerinas. J Strength Conditioning Res 2004; 18: 714-718.

3. Ahn S, Song R. Effects of tai chi exercise on glucose control, neuropathy scores, balance, and quality of life in patients with type 2 diabetes and neuropathy. J Altern Complement Med 2012; 18: 1172-1178.

4. Guo LY, Yang CP, You YL, Chen SK, Yang CH, Hou YY, $\mathrm{Wu}$ WL. Underlying mechanisms of tai-chi-chuan training for improving balance ability in the elders. Chin J Integr Med 2014; 20: 409-415.

5. Jiménez-Martín PJ, Meléndez-Ortega A, Albers U, Schofield D. A review of tai chi chuan and parameters related to balance. Euro J Integr Med 2013; 5: 469-475.

6. Li JX, Xu DQ, Hong Y. Changes in muscle strength, endurance, and reaction of the lower extremities with tai chi intervention. J Biomech 2009; 42: 967-971.

7. Mak MK, Ng PL. Mediolateral sway in single-leg stance is the best discriminator of balance performance for taichi practitioners. Arch Phys Med Rehabil 2003; 84: 683-686.

8. Webster CS, Luo AY, Krageloh C, Moir F, Henning M. A systematic review of the health benefits of tai chi for students in higher education. Prev Med Rep 2016; 3: 103-112.

9. Angioi M, Metsios GS, Koutedakis Y, Wyon MA. Fitness in contemporary dance: A systematic review. Int J Sports Med 2009; 30: 475-484.

10. Lan C, Lai JS, Chen SY, Wong MK. Tai chi chuan to improve muscular strength and endurance in elderly individuals: A pilot study. Arch Phys Med Rehabil 2000; 81: 604-607.

11. Cohen J. A power primer. Psychol Bulletin 1992; 112: 155-159.

12. Mao DW, Hong Y, Li JX. Characteristics of foot movement in tai chi exercise. Phys Ther 2006; 86: 215-222.

13. Wu G, Hitt J. Ground contact characteristics of tai chi gait. Gait Posture 2005; 22: 32-39.

14. Wu G, Liu W, Hitt J, Millon D. Spatial, temporal and muscle action patterns of tai chi gait. J Electromyography Kinesiol 2004; 14: 343-354.

15. Koutedakis Y, Hukam H, Metsios G, Nevill A, Giakas G, Jamurtas A, Myszkewycz L. The effects of three months of aerobic and strength training on selected performance- 
and fitness-related parameters in modern dance students. J Strength Conditioning Res 2007; 21: 808-812.

16. Hamilton WG, Hamilton LH, Marshall P, Molnar M. A profile of the musculoskeletal characteristics of elite professional ballet dancers. Am J Sports Med 1992; 20: 267-273.

17. Li Y, Devault CN, Van Oteghen S. Effects of extended tai chi intervention on balance and selected motor functions of the elderly. Am J Chin Med 2007; 35: 383-391.

18. Xu D, Hong Y, Li J, Chan K. Effect of tai chi exercise on proprioception of ankle and knee joints in old people. Brit J Sports Med 2004; 38: 50-54.

19. Li JX, Hong Y, Chan KM. Tai chi: Physiological characteristics and beneficial effects on health. Brit J Sports Med 2001; 35: 148-156.

20. Twitchett EA, Koutedakis Y, Wyon MA. Physiological fitness and professional classical ballet performance: A brief review. J Strength Conditioning Res 2009; 23: 2732-2740.

21. Zheng G, Lan X, Li M, Ling K, Lin H, Chen L, Fang Q. Effectiveness of tai chi on physical and psychological health of college students: Results of a randomized controlled trial. Plos One 2015; 10: e0132605.

\section{*Correspondence to}

Yung-Shen Tsai

Institute of Sports Equipment Technology

University of Taipei

Taipei

Taiwan 